\title{
Comparison of the Efficacy and Safety of
} Dexmedetomidine Administrated in two Different Modes in Sedative and Analgesic Anesthesia: A Randomized Controlled Trial

\section{Weipeng Xia}

Chinese Academy of Medical Sciences \& Peking Union Medical College

\section{Lingxin Wei}

Chinese Academy of Medical Sciences \& Peking Union Medical College

Xiaoming Deng ( $\nabla$ dengxiaoming2003@sina.com )

Chinese Academy of Medical Sciences \& Peking Union Medical College

\section{Dong Yang}

Chinese Academy of Medical Sciences \& Peking Union Medical College Jinghu Sui

Chinese Academy of Medical Sciences \& Peking Union Medical College

\section{Yulei Sun}

Chinese Academy of Medical Sciences \& Peking Union Medical College

Juhui Liu

Chinese Academy of Medical Sciences \& Peking Union Medical College

\section{Lei Wang}

Chinese Academy of Medical Sciences \& Peking Union Medical College

\section{Research Article}

Keywords: Dexmedetomidine, Sedative and analgesic anesthesia, Loading dose, Hemodynamics, Respiration

Posted Date: December 17th, 2020

DOl: https://doi.org/10.21203/rs.3.rs-118129/v1

License: (9) (1) This work is licensed under a Creative Commons Attribution 4.0 International License. Read Full License 


\title{
Comparison of the efficacy and safety of dexmedetomidine administrated in two different modes in sedative and analgesic anesthesia: a randomized controlled trial
}

\author{
Weipeng Xia ${ }^{1}$, Lingxin $\mathrm{Wei}^{1}$, Xiaoming Deng ${ }^{1 *}$, Dong Yang ${ }^{1}$, Jinghu Sui ${ }^{1}$, Yulei \\ Sun ${ }^{1}$, Juhui Liu ${ }^{1} \&$ Lei Wang ${ }^{1}$ \\ *Correspondence: dengxiaoming2003@sina.com \\ ${ }^{1}$ Department of Anesthesiology, Plastic Surgery Hospital, Chinese Academy of \\ Medical Science \& Peking Union Medical College, Beijing, China
}

\section{Abstract}

Background: To observe and compare the efficacy and safety of dexmedetomidine administrated in two different modes in sedative and analgesic anesthesia.

Methods: Sixty female patients, of American Society of Anesthesiologists physical status I or II, aged from 18-52 years and scheduled for elective plastic operations under sedative and analgesic anesthesia were randomly divided into a control group and an intervention group, with 30 patients in each group. All patients were administered IV midazolam $0.04 \mathrm{mg} / \mathrm{kg}$ and a continuous infusion of $0.1 \mu \mathrm{g} / \mathrm{kg} / \mathrm{min}$ of remifentanil at the beginning of anesthesia. Patients in the control group received IV dexmedetomidine (DEX) $1 \mu \mathrm{g} / \mathrm{kg}$ over $15 \mathrm{~min}$ followed by $0.4-0.7 \mu \mathrm{g} / \mathrm{kg} / \mathrm{h}$ infusion while patients in the intervention group received IV DEX $1 \mu \mathrm{g} / \mathrm{kg}$ over $30 \mathrm{~min}$ followed by $0.4-0.7 \mu \mathrm{g} / \mathrm{kg} / \mathrm{h}$ infusion. Heart rate (HR), mean arterial pressure (MAP), respiratory rate (RR), bispectral index (BIS) and Ramsay sedation scores (RSS) were recorded. The lowest respiratory rates (LRR), incidences of respiratory depression, incidences of oxygen supplementation by facial mask and thrusting the jaw, frequencies of body movements, and additional rescue drug administrations were also recorded. After surgery, recovery time, recall of intraoperative events, pain scores in postanesthesia care unit(PACU), and 
satisfaction of patients and surgeons were assessed.

Results: The BIS at time points from 5 min after anesthesia to the end of surgery in the intervention group were significantly higher than those in the control group $(P<0.05)$. The RSS scores at time points from $5 \mathrm{~min}$ after anesthesia to immediately after induction with DEX were significantly higher in the intervention group than those in the control group $(P<0.05)$. The HR at time points from the beginning of surgery to 30 min after local anesthesia, the MAP at time points from 30 min after local anesthesia to the end of surgery, and the RR at time points from 5 min after anesthesia to the end of surgery were significantly higher in the intervention group than those in the control group $(P<0.05)$. Compared with the control group, patients in the intervention group had higher LRR, lower incidences of respiratory depression, more oxygen supplementation by facial mask and thrusting the jaw, and a shorter recovery time $(P<0.05)$.

Conclusions: The two different dexmedetomidine administrations effectively sedated and provided analgesia for patients. Dexmedetomidine infused with a loading dose over 30 min had less impact on patients' hemodynamics and respiration and could shorten the recovery time after anesthesia.

Trial registration: Registered at the Chinese Clinical Trial Registry, registration number ChiCTR1900027958, date of registration: 07/12/2019.

Key words: Dexmedetomidine; Sedative and analgesic anesthesia; Loading dose; Hemodynamics; Respiration

\section{Background}

Dexmedetomidine (DEX) has been used extensively in various superficial surgical procedures and sedation radiographic tests as it induces sedation, analgesia and anxiolysis without significant respiratory depression [1]. DEX is currently administrated as a loading dose over 10-15 minutes to rapidly achieve a certain blood concentration, followed by a continuous infusion [2]. Due to the limited strength of its sedative and analgesic effects, DEX is often administrated to patients with other 
analgesics or narcotic drugs in sedative and analgesic anesthesia. When used in combination, DEX can improve the anesthetic effect, but may also act to increase the risk of intraoperative respiratory depression [3]. Combination of DEX with remifentanil can induce cardiovascular complications such as a lower heart rate [4]. Previous research reported that omitting the loading dose of DEX avoided haemodynamic side effects without compromising sedation and analgesia [5]. No investigation has yet to evaluate whether the prolonged loading dose time of DEX would produce comparable anesthetic effect and decrease the incidences of side effects. With an emphasis on the quality of anesthesia, we designed this study to determine whether the difference in loading dose infusion time would produce different efficacy and safety in sedative and analgesic anesthesia during plastic surgeries.

\section{Methods}

\section{Patients}

The study protocol was approved by Ethic Committee of Plastic Surgery Hospital, Chinese Academy of Medical Science (2016-12) and written conformed content was obtained from each patient prior to start. The study was conducted in compliance with the recommendations of the CONSORT Statement and the Declaration of Helsinki. The trial was registered at the Chinese Clinical Trial Register, registration number ChiCTR1900027958. A total of 60 female patients, American Society of Anesthesiologists (ASA) physical status class I or II, aged 18-52 years, and scheduled for elective plastic surgery under sedative and analgesic anesthesia were enrolled in this trial. The patients were allocated randomly to one of the two groups $(n=30)$ using a computer-generated list of random numbers. Exclusion criteria were: bradycardia (HR $<50 \mathrm{beat} / \mathrm{min}$ ), hypotension (Mean arterial pressure [MAP] $<60 \mathrm{mmHg}$ ), severe disease (heart, pulmonary, hepatic or renal), obesity (Body mass index $[\mathrm{BMI}] \geq 30$ ), obstructive sleep apnea/hypopnea syndrome, and known allergy to DEX.

\section{Monitoring indicators}

No premedication was given to any of the patients. After being taken to the operating room, standard monitoring included electrocardiogram (ECG), noninvasive blood 
pressure (BP), heart rate (HR) and oxyhemoglobin saturation $\left(\mathrm{SpO}_{2}\right)($ Datex-Ohmeda Division, Instrumentarium Corp., Helsinki, Finland). Respiratory rate (RR) was monitored by a nasal end-tidal $\mathrm{CO}_{2}$ cannula. The bispectral index (BIS) was also continuously monitored (BIS Vista, Covidien, Boulder, CO, USA). A peripheral intravenous (IV) catheter was inserted by nurse.

\section{Anesthesia management}

All patients received an IV of midazolam $(0.04 \mathrm{mg} / \mathrm{kg})$ and a continuous infusion of remifentanil $(0.1 \mu \mathrm{g} / \mathrm{kg} / \mathrm{min})$ at the beginning of anesthesia. Patients in the control group received IV DEX $1 \mu \mathrm{g} / \mathrm{kg}$ over $15 \mathrm{~min}$ followed by $0.4-0.7 \mu \mathrm{g} / \mathrm{kg} / \mathrm{h}$ infusion and patients in the intervention group received IV DEX $1 \mu \mathrm{g} / \mathrm{kg}$ over $30 \mathrm{~min}$ followed by 0.4-0.7 $\mu \mathrm{g} / \mathrm{kg} / \mathrm{h}$ infusion. During the surgery, the level of sedation status was assessed using the Ramsay Sedation Scores (RSS) [6] (Table 1) and the BIS. The goal of sedation was to achieve an RSS of 3-5 or BIS of $60-80$. Therefore, local anesthesia was performed. The patients' MAP and HR were maintained at a range of baseline $\pm 20 \%$. Atropine $(0.3 \mathrm{mg})$ and ephedrine $(6 \mathrm{mg})$ were intravenously administrated for bradycardia (HR $<50$ beats/min for $>60 \mathrm{~s}$ ) and hypotension (MAP $<60 \mathrm{mmHg}$ for $>$ $60 \mathrm{~s})$, respectively. A facial oxygen mask $(6 \mathrm{~L} / \mathrm{min})$ was used for hypoxia $\left(\mathrm{SpO}_{2}<90 \%\right)$. If airway obstruction occurred, the patient was treated by thrusting the jaw. Midazolam ( $2 \mathrm{mg}$ ) was administrated as a rescue drug when the infusion rate of DEX was increased to a maximum of $0.7 \mu \mathrm{g} / \mathrm{kg} / \mathrm{h}$ and the depth of sedation was not achieved. The infusion of DEX was stopped $30 \mathrm{~min}$ before the end of surgery, and the infusion of remifentanil was turned off at the end of the surgery.

\section{Outcome measurements}

HR, MAP, RR, BIS, RSS were evaluated and recorded before anesthesia (baseline) $\left(\mathrm{T}_{0}\right)$, 5 min after anesthesia $\left(T_{1}\right), 10$ min after anesthesia $\left(T_{2}\right)$, immediately after induction with $\operatorname{DEX}\left(\mathrm{T}_{3}\right)$, the beginning of local anesthesia $\left(\mathrm{T}_{4}\right)$, the beginning of surgery $\left(\mathrm{T}_{5}\right)$, 30 min after local anesthesia $\left(\mathrm{T}_{6}\right), 60 \mathrm{~min}$ after local anesthesia $\left(\mathrm{T}_{7}\right)$, immediately after turning off DEX infusion ( $\left.\mathrm{T}_{8}\right)$, and the end of surgery $\left(\mathrm{T}_{9}\right)$. The lowest respiratory rates (LRR), incidences of respiratory depression $(\mathrm{RR}<10$ breaths $/ \mathrm{min})$, incidences of 
oxygen supplementation by facial mask and thrusting the jaw, frequencies of body movements, and additional rescue drug administrations were also recorded throughout the procedure. After surgery, recovery time, recall of intraoperative events, and pain scores using a numerical pain scale $(0=$ no pain to $10=$ the worst pain) [7] in PACU were assessed. The satisfaction of patients and surgeons with the anesthesia were assessed using a 5-point numerical rating scale [8] (Table 1).

\section{Table 1. Satisfaction scale and Ramsay sedation scores}

\begin{tabular}{ll}
\hline Satisfaction scale \\
0 & Extremely dissatisfied \\
1 & Dissatisfied \\
2 & Neutral \\
3 & Satisfied \\
4 & Extremely satisfied \\
& \\
Ramsay sedation scores \\
1 & Anxious and agitated, restless \\
2 & Cooperative, oriented, tranquil \\
3 & Responsive to verbal commands, drowsy \\
4 & Asleep, responsive to light stimulation \\
5 & Asleep, slow response to stimulation \\
6 & No response to stimulation \\
\hline
\end{tabular}

\section{Statistical analysis}

Calculation of sample size was based on the primary endpoint of BIS value immediately after induction with DEX (T3). In our pilot study, the BIS value (T3) in the control group was 65.9 \pm 5.72 . A power analysis with a two-sided significance alpha level of 0.05 and a power of 0.8 indicated that a sample size of 21 patients in each group was enough to detect a 5 difference in BIS value after induction with DEX. We aimed to recruit 30 patients in each group to account for the possible exclusions and dropouts.

All statistical analyses were performed using SPSS 17.0 (SPSSFW, SPSS Inc.). The normality of distribution was assessed with the Shapiro-Wilk test. Normally distributed data are presented as mean $\pm \mathrm{SD}$, and skewed data were presented as a median with an interquartile range [IQR]). Independent t-test was used to examine the difference of normally distributed continuous variables between groups. Skewed data were analyzed by Mann-Whitney U-test. Categorical data are presented by frequency and analyzed by $\chi^{2}$ test. A $P$ value of $<0.05$ was considered statistically significant. 


\section{Results}

The procedure was successfully performed in each of the 60 patients recruited in the study. In the end, all of the 60 patients (30 in the control group and 30 in the intervention group) were included in the final analysis (Figure 1). No significant differences were observed in patient demographic data including gender, age, ASA classification and BMI (Table 2). The surgery time did not differ between groups.

Figure 2 and Table 3 show the changes in sedation level during the study. Patients in the intervention group had higher BIS at time points from 5 min after anesthesia to the end of surgery $(P<0.05)$. Patients in the intervention group also had higher RSS scores compared with the control group at time points from 5 min after anesthesia to immediately after induction with $\operatorname{DEX}(P<0.05)$.

Figure 3 displays the hemodynamic and respiratory parameters changes during the study. Patients in the intervention group had higher HR at time points from the beginning of surgery to $30 \mathrm{~min}$ after local anesthesia, higher MAP at time points from 30 min after local anesthesia to the end of surgery, and higher RR at time points from 5 min after anesthesia to the end of surgery $(P<0.05)$.

Compared with the control group, patients in the intervention group had higher LRR, lower incidences of respiratory depression, lower incidences of oxygen supplementation by facial mask and thrusting the jaw, and a shorter recovery time $(P<0.05)$ (Table 4).

No significant differences were found in body movements, additional rescue drug administrations, recall of intraoperative events, pain scores and the satisfaction levels of patients and surgeons between groups $(P>0.05)$ (Table 4$)$.

Table 2. Patients' demographic data and surgery time

\begin{tabular}{lccc}
\hline & Control Group & Intervention Group & P value \\
\hline Gender (M/F) & $0 / 30$ & $0 / 30$ & 1.000 \\
Age (yrs) & $32.2 \pm 8.4$ & $30.2 \pm 6.3$ & 0.301 \\
BMI (kg/m ${ }^{2}$ ) & $21.5 \pm 2.6$ & $20.4 \pm 2.4$ & 0.075 \\
ASA (I/II) & $30 / 0$ & $30 / 0$ & 1.000 \\
Surgery time (min) & $123.0 \pm 37.9$ & $125.0 \pm 64.9$ & 0.888 \\
\hline
\end{tabular}

Values are mean $\pm \mathrm{SD}$ or number 
Table 3. Ramsay sedation scores

\begin{tabular}{cccc}
\hline & Control Group & Intervention Group & $P$ value \\
\hline T0 & $2(2-2)$ & $2(2-2)$ & 1.000 \\
T1 & $3(2-3)$ & $2(2-2)^{*}$ & 0.000 \\
T2 & $4(3-4)$ & $3(3-3)^{*}$ & 0.000 \\
T3 & $4(4-4)$ & $4(4-4)^{*}$ & 0.010 \\
T4 & $4(4-4)$ & $4(4-4)$ & 0.133 \\
T5 & $4(4-4)$ & $4(4-4)$ & 0.317 \\
T6 & $4(4-4)$ & $4(4-4)$ & 0.305 \\
T7 & $4(4-4)$ & $4(4-4)$ & 0.642 \\
T8 & $4(3.75-4)$ & $4(4-4)$ & 0.153 \\
T9 & $4(3-4)$ & $4(3-4)$ & 0.624 \\
\hline
\end{tabular}

Values are medians (interquartile range)

$* P<0.05$ compared with the Control group

Table 4. Assessments of intraoperative events and evaluations in PACU

\begin{tabular}{lccc}
\hline & Control Group & Intervention Group & $\boldsymbol{P}_{\mathbf{v}}$ \\
\hline Lowest respiratory rate (breaths/min) & $7.1 \pm 2.5$ & $8.6 \pm 1.8^{*}$ & 0. \\
Respiratory depression & $24(80.0)$ & $15(50.0)^{*}$ & 0. \\
Incidences of & & & \\
$\quad$ oxygen supplementation by facial mask & $24(80.0)$ & $7(23.3)^{*}$ & 0. \\
$\quad$ thrusting the jaw & $11(36.7)$ & $1(3.3)^{*}$ & 0. \\
$\quad$ recall of events during surgery & $0(0)$ & $0(0)$ & 1. \\
Frequencies of & & & 0.0 .0 \\
$\quad$ body movements & $0(0-0)$ & $1(0-2)$ & 0. \\
$\quad$ additional rescue drug administrations & $1(0.75-2)$ & $16.0 \pm 3.0^{*}$ & 0. \\
Recovery time (min) & $22.9 \pm 9.6$ & $0(0-1)$ & 0. \\
VAS score & $0(0-1)$ & $4(4-4)$ & 0. \\
Satisfaction score of patients & $4(4-4)$ & $4(4-4)$ & 0. \\
Satisfaction score of surgeons & $4(4-4)$ & & \\
\hline
\end{tabular}

Values are mean \pm SD or medians (interquartile range)

$* P<0.05$ compared with the Control group 


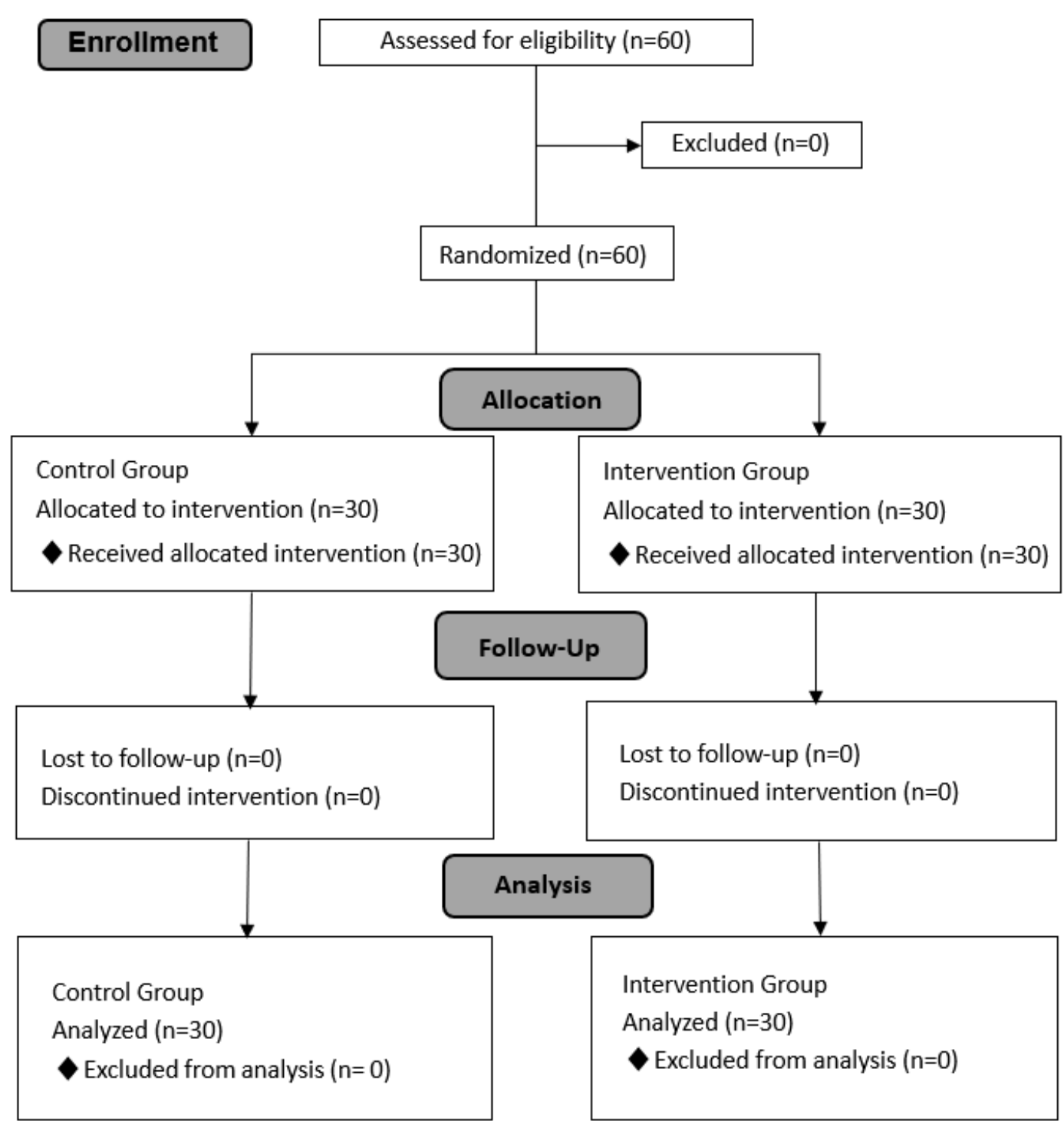

Figure1. A flow diagram shows the patients recruitment.

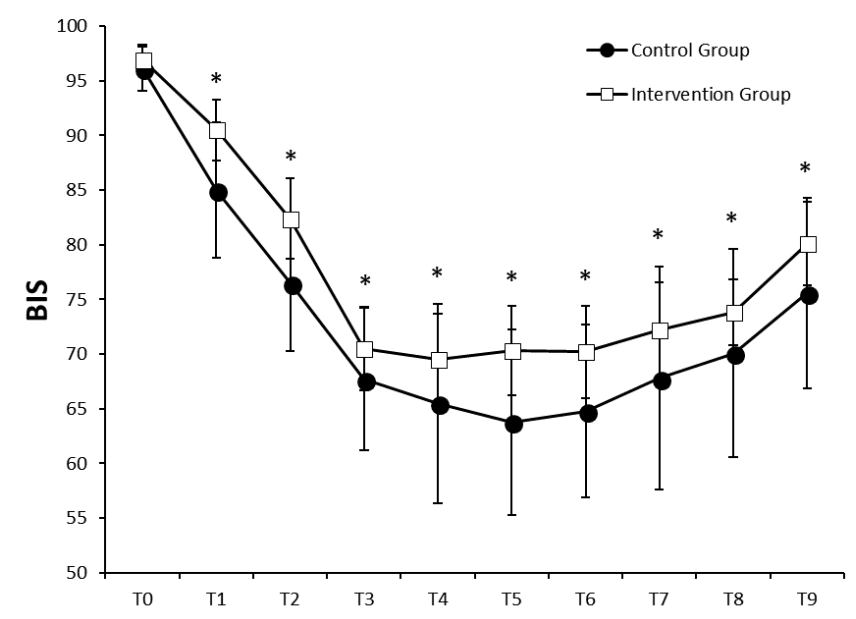

Figure 2. Changes in BIS. $* P<0.05$ compared with the Control group. 

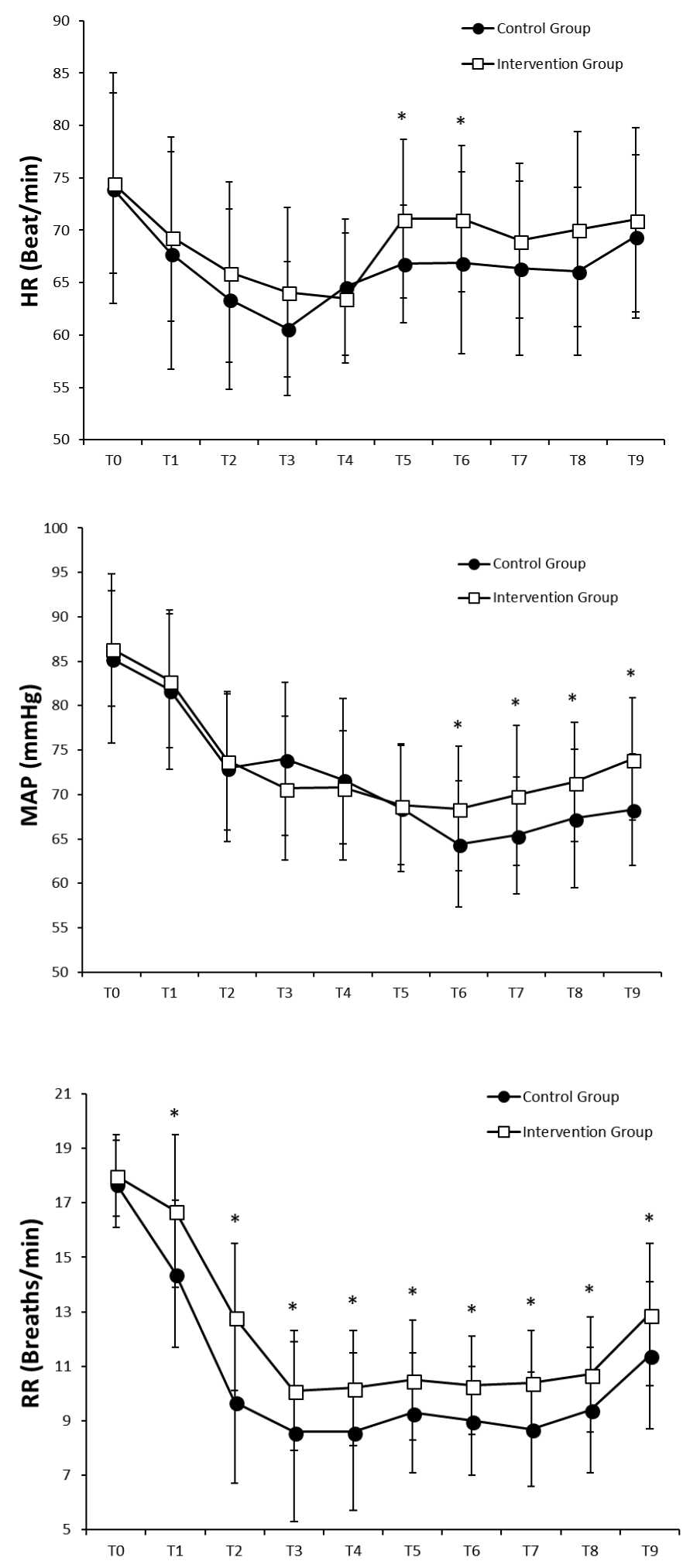

Figure 3. Changes in HR, MAP and RR. ${ }^{*} P<0.05$ compared with the Control group. 


\section{Discussion}

In this study, we found that although DEX infused with a loading dose over 15 min provided deeper sedation than DEX infused with a loading dose over $30 \mathrm{~min}$, these two different DEX administrations could both be effectively applied in the surgery. In addition, DEX infused with a loading dose over 30 min resulted in more stable hemodynamics, less respiratory depression, and shorted recovery time for patients in sedative and analgesic anesthesia.

In order to achieve satisfactory sedation and analgesia, DEX is often used clinically in combination with other anesthetic drugs such as midazolam and opioids [9-11]. Midazolam reduces the incidence of recall of intraoperative events by producing anterograde amnesia [12]. Studies have shown that DEX combined with midazolam provided better sedation than midazolam alone in patients undergoing fiberoptic bronchoscopy [13]. The analgesic effect of DEX combined with opioids is beneficial in invasive operations. For example, a previous study demonstrated dexmedetomidine combined with remifentanil provided better analgesia and higher satisfaction levels amongst surgeons than midazolam combined with remifentanil in patients undergoing radiofrequency ablation for atrial fibrillation [8]. In our study, DEX was administrated with midazolam and remifentanil to produce sufficient sedation and analgesia for the surgery.

While enhancing the anesthetic effect, the combination of sedation and analgesic drugs may also increase the incidences of respiratory and circulatory depressions. Clinically, the safety of anesthesia can be improved by reducing the dose of drugs or adjusting the method of drug administration [14-16]. In our study, we extended the infusion time of DEX loading dose from $15 \mathrm{~min}$ to $30 \mathrm{~min}$ to delay the increment of DEX plasma concentration and attenuate the DEX peak plasma concentration. As shown in the present study, patients in the intervention group had a more gradual BIS change after DEX infusion. However, no differences were found between the groups in RSS from the beginning of local anesthesia to the end of surgery, intraoperative body movements, additional rescue drug administrations, recall of intraoperative events, pain scores in 
PACU, or the satisfaction levels of patients and surgeons. Our results revealed that although the sedation depth in the intervention group was relatively shallow, this mode of DEX administration could achieve the sedation and analgesia required for the surgery. In a study of sedative and analgesic anesthesia, use of dexmedetomidine could significantly prolong postoperative recovery time [17]. In this study, the DEX plasma concentration of the intervention group after the loading dose infusion was theoretically lower than that of the control group. We found no differences in the surgery time between groups which lead to a better early postoperative recovery for patients in the intervention group.

With respect to hemodynamic stability, Bloor et al. [18] reported that the amount of DEX loading dose and the duration of administration affected the blood pressure, with blunting of the blood pressure by $23 \% 60 \mathrm{~min}$ after infusion of $1 \mu \mathrm{g} / \mathrm{kg}$ DEX over 2 min. The remifentanil used in this study is a non-accumulative, ultra-short-acting opioid which allows faster recovery after anesthesia [19, 20]. We know from earlier studies that both remifentanil and DEX reduce HR [21, 22]. We observed that the intervention group had higher MAP at time points from 30 min after local anesthesia to the end of surgery and higher HR at time points from the beginning of surgery to 30 min after local anesthesia, which indicated that prolonged DEX loading dose time contributed to more stable hemodynamics.

Though DEX provides sedation and analgesia without significant respiratory depression, respiratory changes needed to be measured in the case of drug combinations [23]. The depth of sedation can significantly affect patients' minute ventilation and RR [24]. In the present study, we found both groups showed respiratory depression during anesthesia. However, the intervention group had higher RR throughout the study and also had higher LRR, lower incidences of respiratory depression, oxygen supplementation by facial mask and thrusting the jaw. We concluded that prolonged DEX loading dose time reduced adverse respiratory reactions.

There are a few limitations to this study. The method of DEX administration in the control group is extensively applied in clinical practice. Though higher respiratory depression was observed in this group, the problem could be solved by oxygen supplementation with facial mask or thrusting the jaw. Before the study, we excluded 
patients with known obesity or obstructive sleep apnea/hypopnea syndrome. For patients with a predictable risk of respiratory depression, further research is needed to identify the safety of this method of DEX administration.

\section{Conclusion}

In conclusion, our study demonstrated that DEX is an excellent drug when combined with other narcotic drugs in sedative and analgesic anesthesia. Prolonging the DEX loading dose infusion time from $15 \mathrm{~min}$ to $30 \mathrm{~min}$ can provide sufficient sedation during the surgery with more stable hemodynamics, as well as less respiratory adverse reactions.

\section{Abbreviations}

DEX: Dexmedetomidine

HR: Heart rate

MAP: Mean arterial pressure

RR: Respiratory rate

BIS: Bispectral index

RSS: Ramsy sedation scores

LRR: Lowest respiratory rates

PACU: Postanesthesia care unit

ASA: American Society of Anesthesiologists

BMI: Body mass index

ECG: Electrocardiogram

\section{Acknowledgements}


The authors thank the anesthetists and surgeons at our hospital who participated in this study. We would also like to acknowledge the professional assistance provided by Gilbert Xavier Gonzalez (Institute for Biomedical Sciences, Georgia State University, Atlanta, GA, USA) in reviewing and editing the English grammar in this manuscript.

\section{Authors' Contributions}

WPX, LXW and JHS designed and conducted the study, interpreted data and drafted the manuscript. WPX, DY, LW, JHL and YLS revised the manuscript and did the statistical analysis. XMD revised the manuscript. All authors read and approved the final manuscript.

\section{Founding}

This study was supported by Institutional Foundation of Plastic Surgery Hospital (No. Z2015005).

\section{Availability of data and materials}

The datasets used and analyzed during the current study are available from the corresponding author on reasonable request.

\section{Ethics approval and consent to participate}

The study protocol was approved by Ethic Committee of Plastic Surgery Hospital, Chinese Academy of Medical Science (2016-12). All procedures were performed according to the Declaration of Helsinki and written conformed content was obtained from each patient prior to start. The trial was registered at the Chinese Clinical Trial Registry, registration number ChiCTR1900027958.

\section{Consent for publication}

Not applicable. 


\section{Competing interests}

The authors declare that they have no competing interests.

\section{Authors' information}

${ }^{1}$ Department of Anesthesiology, Plastic Surgery Hospital, Chinese Academy of Medical Science \& Peking Union Medical College, 33 Badachu Rd, Shijingshan District, Beijing 100144, China

Email:

Weipeng Xia: xiaweipeng0428@163.com

Lingxin Wei: weilx3934@ sina.com

Xiaoming Deng: dengxiaoming2003@sina.com

Dong Yang: yangdonglin28@126.com

Jinghu Sui: mazui@126.com

Yulei Sun: sunyulei@hotmail.com

Juhui Liu: liujuhui2006@126.com

Lei Wang: 100wlwl@163.com

\section{Corresponding author}

Correspondence to Xiaoming Deng

Department of Anesthesiology, Plastic Surgery Hospital, Chinese Academy of Medical Science \& Peking Union Medical College, Beijing, China

Email: dengxiaoming2003@sina.com 


\section{References}

1. Candiotti KA, Bergese SD, Bokesch PM, Feldman MA, Wisemandle W, Bekker AY. Monitored anesthesia care with dexmedetomidine: a prospective, randomized, doubleblind, multicenter trial. Anesth Analg. 2010;110(1):47-56.

2. Gerlach AT, Dasta JF. Dexmedetomidine: an updated review. Ann Pharmacother. 2007;41(2):245-52.

3. Gertler R, Brown HC, Mitchell DH, Silvius EN. Dexmedetomidine: a novel sedativeanalgesic agent. Proc (Bayl Univ Med Cent). 2001;14(1):13-21.

4. Kim N, Yoo Y-C, Lee SK, Kim H, Ju HM, Min KT. Comparison of the efficacy and safety of sedation between dexmedetomidine-remifentanil and propofol-remifentanil during endoscopic submucosal dissection. World J Gastroenterol. 2015;21(12):36718.

5. Ickeringill $M$, Shehabi $Y$, Adamson $H$, Ruettimann U. Dexmedetomidine infusion without loading dose in surgical patients requiring mechanical ventilation: haemodynamic effects and efficacy. Anaesth Intensive Care. 2004;32(6):741-5.

6. Reschreiter $\mathrm{H}$, Maiden $\mathrm{M}$, Kapila A. Sedation practice in the intensive care unit: a UK national survey. Crit Care. 2008;12(6):R152.

7. Farrar JT, Young JP, LaMoreaux L, Werth JL, Poole RM. Clinical importance of changes in chronic pain intensity measured on an 11-point numerical pain rating scale. Pain. 2001;94(2):149-58.

8. Cho JS, Shim J-K, Na S, Park I, Kwak YL. Improved sedation with dexmedetomidine-remifentanil compared with midazolam-remifentanil during catheter ablation of atrial fibrillation: a randomized, controlled trial. Europace. 2014;16(7):1000-6.

9. Heard C, Burrows F, Johnson K, Joshi P, Houck J, Lerman J. A comparison of dexmedetomidine-midazolam with propofol for maintenance of anesthesia in children undergoing magnetic resonance imaging. Anesth Analg. 2008;107(6):1832-9.

10. Nie Y, Liu Y, Luo Q, Huang S. Effect of dexmedetomidine combined with sufentanil for post-caesarean section intravenous analgesia: a randomised, placebo-controlled study. Eur J Anaesthesiol. 2014;31(4):197-203.

11. Feng C, Qi S-h, Zou Y-m, Ma X-s, Gao D-p, Han B-q. [Effects of dexmedetomidine combined with fentanyl in patients undergoing anesthesia induction by sevoflurane]. Zhonghua Yi Xue Za Zhi. 2012;92(27):1889-91.

12. Boku $A$, Inoue $M$, Hanamoto $H$, Oyamaguchi $A$, Kudo $C$, Sugimura $M$, et al. Effective Dosage of Midazolam to Erase the Memory of Vascular Pain During Propofol Administration. Anesth Prog.63(3):147-55.

13. Bergese SD, Patrick Bender S, McSweeney TD, Fernandez S, Dzwonczyk R, Sage $\mathrm{K}$. A comparative study of dexmedetomidine with midazolam and midazolam alone for sedation during elective awake fiberoptic intubation. J Clin Anesth. 2010;22(1):35-40. 14. Siobal MS, Kallet RH, Kivett VA, Tang JF. Use of dexmedetomidine to facilitate extubation in surgical intensive-care-unit patients who failed previous weaning attempts following prolonged mechanical ventilation: a pilot study. Respir Care. 2006;51(5):492-6.

15. Ramaswamy SS, Parimala B. Comparative evaluation of two different loading doses of dexmedetomidine with midazolam-fentanyl for sedation in vitreoretinal surgery under peribulbar anaesthesia. Indian J Anaesth. 2016;60(2):89-93.

16. Jiang W, Wang $Q$, Xu M, Li Y, Yang R, Song X, et al. Assessment of different loading doses of dexmedetomidine hydrochloride in preventing adverse reaction after combined spinal-epidural anesthesia. Exp Ther Med. 2017;13(6):2946-50. 
17. Tosun Z, Akin A, Guler G, Esmaoglu A, Boyaci A. Dexmedetomidine-ketamine and propofol-ketamine combinations for anesthesia in spontaneously breathing pediatric patients undergoing cardiac catheterization. J Cardiothorac Vasc Anesth. 2006;20(4):515-9.

18. Bloor BC, Ward DS, Belleville JP, Maze M. Effects of intravenous dexmedetomidine in humans. II. Hemodynamic changes. Anesthesiology. 1992;77(6):1134-42.

19. Won YJ, Yoo JY, Chae YJ, Kim DH, Park SK, Cho HB, et al. The incidence of postoperative nausea and vomiting after thyroidectomy using three anaesthetic techniques. J Int Med Res. 2011;39(5):1834-42.

20. Beecroft $\mathrm{CL}$, Enright SM, O'Beirne HA. Remifentanil in the management of severe tetanus. Br J Anaesth. 2005;94(1):46-8.

21. Bhana N, Goa KL, McClellan KJ. Dexmedetomidine. Drugs. 2000;59(2).

22. Reid JE, Mirakhur RK. Bradycardia after administration of remifentanil. $\mathrm{Br} \mathrm{J}$ Anaesth. 2000;84(3):422-3.

23. Palanisamy A, Klickovich RJ, Ramsay M, Ouyang DW, Tsen LC. Intravenous dexmedetomidine as an adjunct for labor analgesia and cesarean delivery anesthesia in a parturient with a tethered spinal cord. Int J Obstet Anesth. 2009;18(3):258-61.

24. Deitch K, Miner J, Chudnofsky CR, Dominici P, Latta D. Does end tidal CO2 monitoring during emergency department procedural sedation and analgesia with propofol decrease the incidence of hypoxic events? A randomized, controlled trial. Ann Emerg Med. 2010;55(3):258-64. 


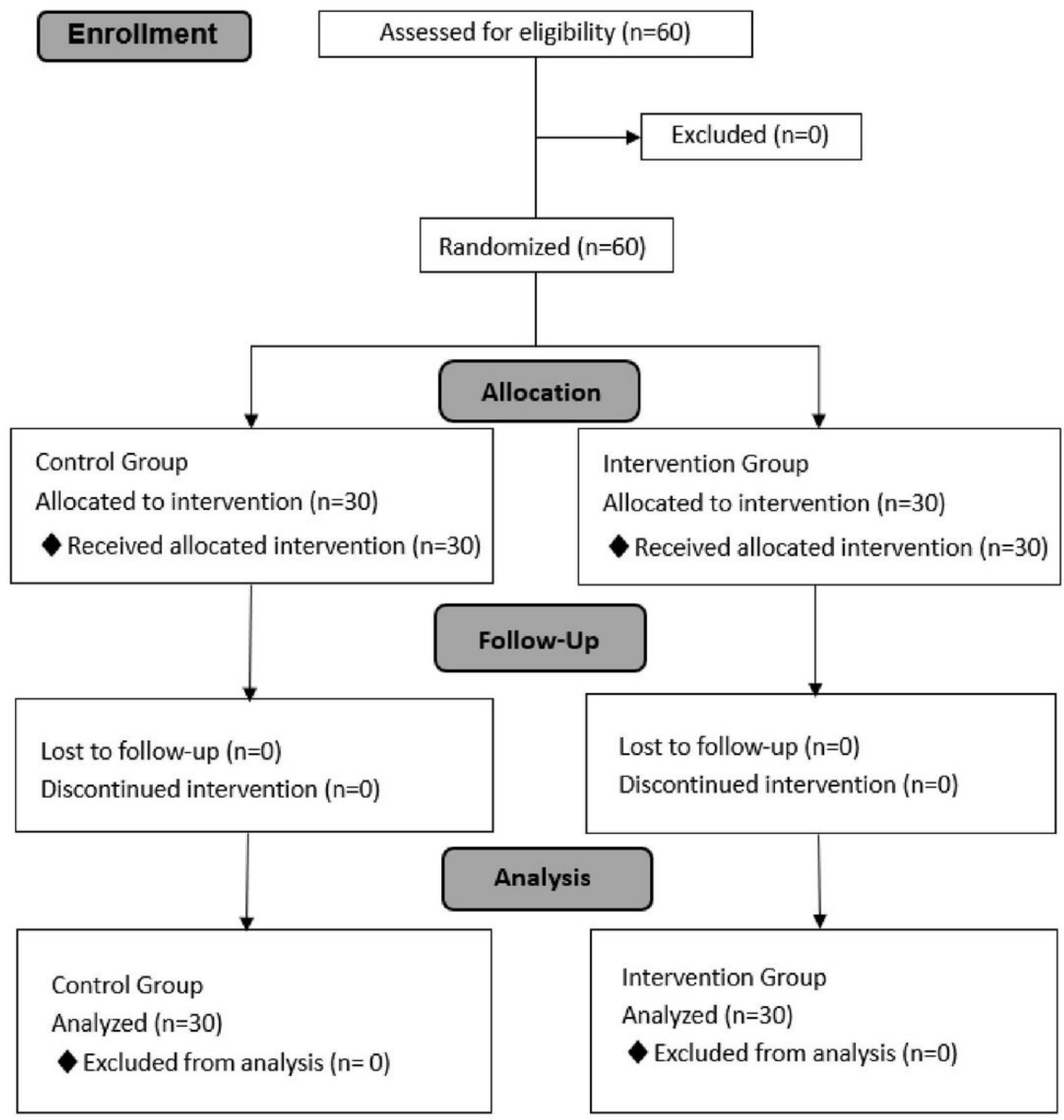

Figure 1

A flow diagram shows the patients recruitment. 


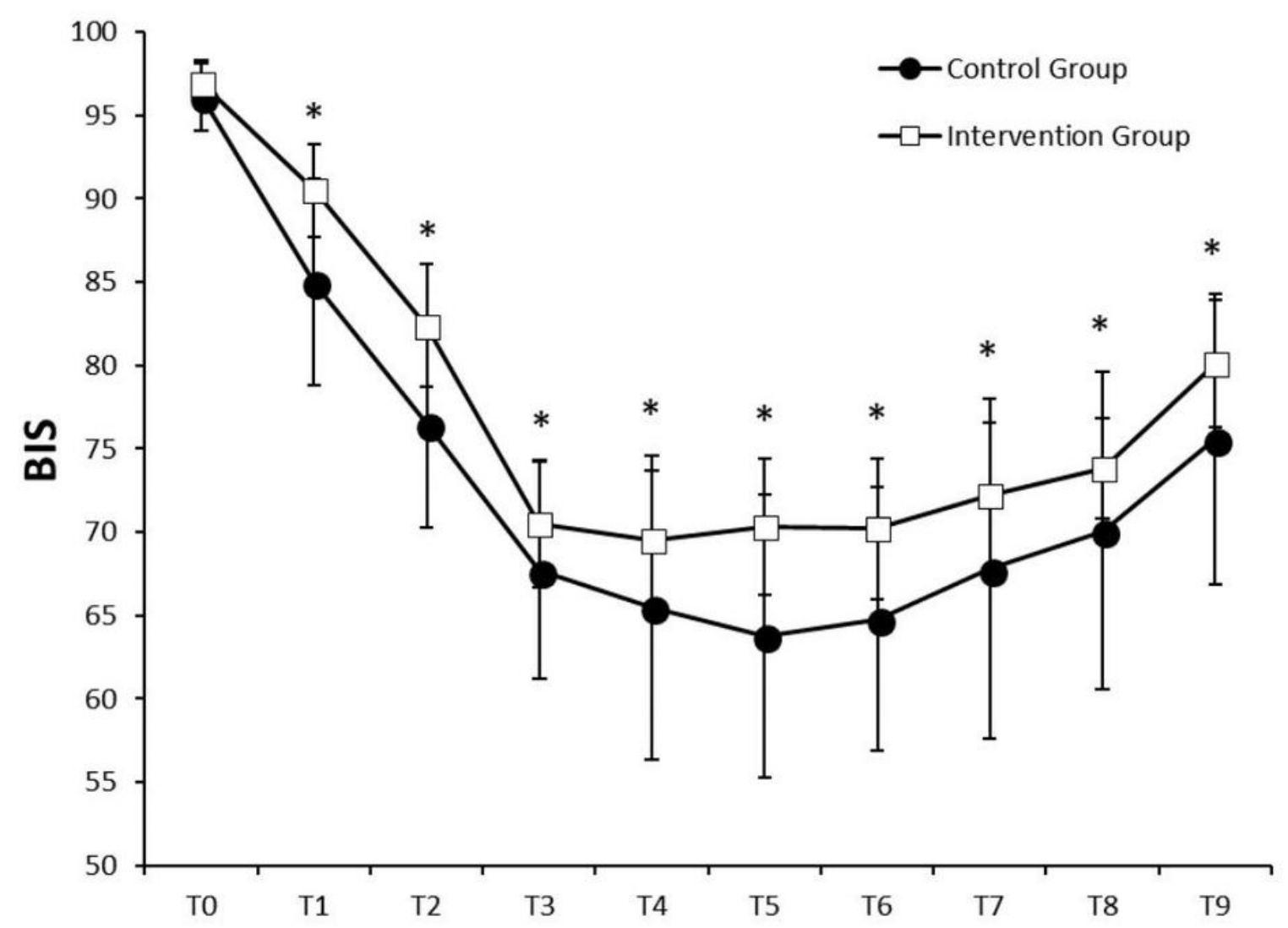

Figure 2

Changes in BIS. ${ }^{*} \mathrm{P} \otimes 0.05$ compared with the Control group. 

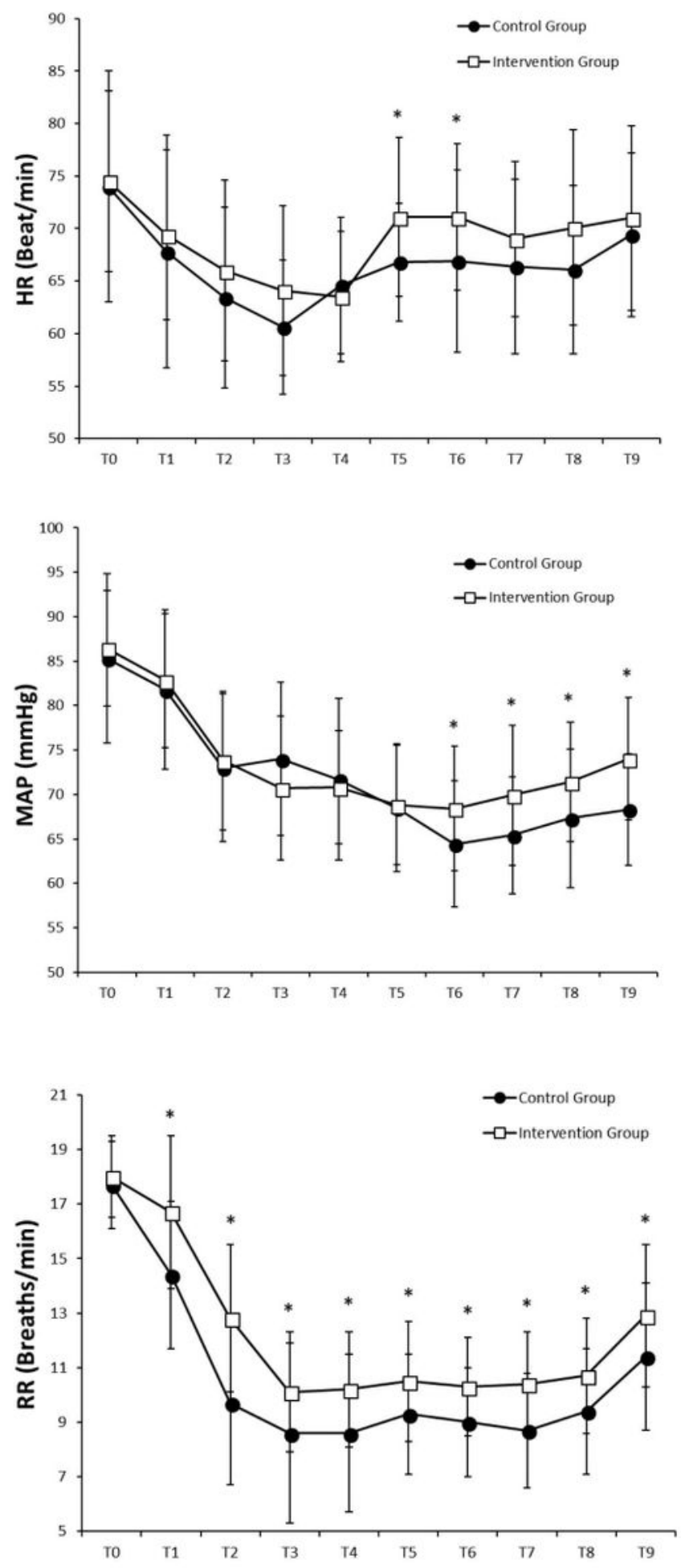

Figure 3

Changes in HR, MAP and RR. *P冈0.05 compared with the Control group. 